\title{
Effects of Alkali Metallization on the Luminescence Degradation of Porous Silicon
}

\author{
E. KAYAHAN ${ }^{a, b}$, M. ÖZER ${ }^{c}$ AND A.Y. ORAL ${ }^{d}$ \\ ${ }^{a}$ Kocaeli University, Gebze MYO, 41420, Çayırova, Kocaeli, Turkey \\ ${ }^{b}$ Natural and Applied Sci. Inst. Electro-optic and Sys. Eng. Umuttepe, Kocaeli University \\ 41380, Kocaeli, Turkey \\ ${ }^{c}$ Physics Dep., Istanbul Kültür University, 34136, Bakırköy, Istanbul, Turkey \\ ${ }^{d}$ Materials Sci. Eng., Gebze Institute of Technology, 41400, Çayırova, Kocaeli, Turkey
}

\begin{abstract}
In this study, change in the intensity and stability of photoluminescence obtained by porous silicon were investigated with illumination time and metallization state. The porous silicon samples were metallized by immersing into solutions containing $3 \mathrm{mM} \mathrm{LiNO}_{3}, \mathrm{KNO}_{3}$ and $\mathrm{NaNO}_{3}$ metal salts using immersing plating method. The surface bond configurations of porous silicon were monitored by the Fourier transmission infrared spectroscopy and the results showed that the surfaces of the samples were oxidized after the metallization. The photoluminescence intensity increased after certain critical immersion times and photoluminescence spectrum shifted towards the high energy region after the metallization. Photoluminescence intensity of metallized porous silicon samples was more stable than as-anodized porous silicon samples. The experimental results suggested the possibility that oxygen and/or alkali metal ( $\mathrm{Li}, \mathrm{K}$ and $\mathrm{Na}$ ) passivation of porous silicon surface could be a suitable way to obtain an efficient and stabilized photoluminescence.
\end{abstract}

PACS: 78.55.Ap, 78.55.Mb

\section{Introduction}

In recent years, the discovery of strong room temperature photoluminescence (PL) of etched porous silicon (PS) has attracted a vast attention due to its potential applications in Si-based optoelectronic devices [1]. Porous silicon based optoelectronic devices undergo substantial degradation in the luminescence spectrum with aging due to atmospheric oxidation [2]. Therefore, the surface of the PS needs to be passivated to produce a stable luminescence $[2,3]$.

In our previous studies [2-4], the metallization and aging effects on surface properties and PL properties of porous silicon were investigated. Therefore, this study presents the results on the effects of surface modification on the luminescence degradation properties of the PS by adsorption of the $\mathrm{Li}, \mathrm{K}$ and $\mathrm{Na}$ atoms using immersion plating method. The effects of the metallization on photoluminescence properties of the samples were discussed and results of the spectral studies (scanning electron microscopy (SEM), PL and Fourier transform infrared (FTIR)) were reported.

\section{Experimental}

The PS samples were prepared from $p$-type, B doped, (111) oriented silicon wafers with $10.5-19.5 \Omega$ cm resistivity. The porous layers were obtained by anodization in solutions of $\mathrm{HF}(48 \%): \mathrm{C}_{2} \mathrm{H}_{5} \mathrm{OH}(98 \%)=1: 4$ (by volume) and $\operatorname{HF}(48 \%)$ :DI water $=1: 4$ (by volume) for various anodisation times between 10 and 60 min. After the etching, PS samples were rinsed in ethanol and/or DI water, and then dried in vacuum. Afterwards, PS samples were metallized for various times (3-60 s) in solutions containing $3 \mathrm{mM} \mathrm{LiNO}{ }_{3}, \mathrm{KNO}_{3}$ and $\mathrm{NaNO}_{3}$ metal salts by using immersing plating method.

Chemical bonds existing in PS samples before and after the metallization were analyzed by using a FTIR (Shimadzu 8201/86601). The PL spectrum studies were performed by using a PC controlled monolithic miniature spectrometer (MMS-Carl Zeiss). All PL spectra with 305-1150 nm range were measured with 14 bit resolution and $10 \mathrm{~ms}$ minimal integration time. The PL excitation was obtained with a UV lamp set (Konrad-Benda) with wavelengths of 254 and $366 \mathrm{~nm}$. All spectral measurements were taken in air at room temperature.

\section{Result and discussion}

Figure 1 shows SEM micrographs of as-prepared and Na-metallized PS surface. When SEM images of as-anodized PS sample is compared to SEM image of Na-metallized PS, it is clear that Na metals coat PS surface and fill the pores.

The quenching of the PS luminescence of as-anodized PS due to continuous exposure to UV lamp $(365 \mathrm{~nm})$ 


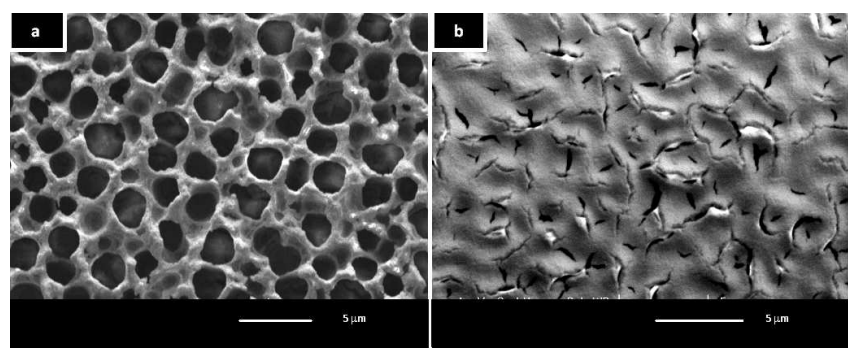

Fig. 1. SEM images of PS surface: (a) as-anodized and (b) Na-metallized PS.

for $20 \mathrm{~min}$ is depicted in Fig. 2. The PL measurements were performed just after the PS fabrication by using the MMS spectrometer.

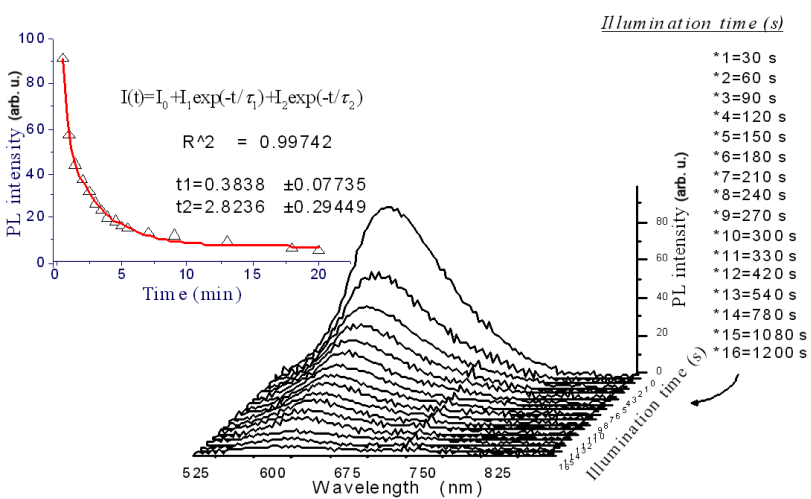

Fig. 2. Illumination time dependence of the photoluminescence spectrum of as-anodized PS under influence of UV illumination at $366 \mathrm{~nm}$. Inset figure shows the variation of $\mathrm{PL}$ intensity with the illumination times. The solid line in inset figure corresponds to exponential function fit.

It is observed that PL intensity of PS rapidly decreases with illumination time (Fig. 2). The decline is so fast that about $80 \%$ PL intensity vanished compared with initial state after $4 \mathrm{~min}$. Peak of PL spectrum also shifts towards high energy region during the illumination. The decrease of PL spectrum is not symmetric around the peak. Full width half maximum (FWHM) of the PL spectrum does not change significantly with the illumination time. Therefore, it may be concluded that the surface states of PS plays an essential role in the quenching of the spectrum during the UV illumination.

It should be noted that the PL signal intensities in Fig. 2 undergo rapid degradation in the first $10 \mathrm{~min}$. Afterwards, the degradation rate constantly slows down. PL intensity decreases with all illumination time. The decrease could be represented with combination of two exponential decays. Empirical relationship for illumination time $(t)$ dependence of the PL intensity is fitted by

$$
I(t)=I_{0}+I_{1} \exp \left(-t / \tau_{1}\right)+I_{2} \exp \left(-t / \tau_{2}\right),
$$

where $I_{0}, I_{1}$ and $I_{2}$, are fitting parameters, $\tau_{1}$ and $\tau_{2}$ are fast and slow decay times of exponential decay in PL spectrum intensity, respectively. It may be concluded that fast and slow degradation observed in PL spectrum (Fig. 2) can be related to photoinduced $\mathrm{H}$ desorption and simultaneous surface oxidation, respectively. The blue shift of PL spectrum under the UV illumination may be described by mechanisms between electron traps localized at $\mathrm{Si}=\mathrm{O}$ bonds at the $\mathrm{Si} / \mathrm{SiO}_{2}$ interface and the free holes in the valence band. On the other hand, gradual reduction in the (nano) crystal size could be attributed to increasing oxidation with increasing the illumination time $[5,6]$.

The PS surface modification due to the metallization was monitored by FTIR spectroscopy. Figure 3a shows the FTIR absorption spectra before and after adsorption of $\mathrm{Na}$ metals on the PS surface by immersion plating in $3 \mathrm{mM} \mathrm{NaNO}$ containing aqueous solution. The spectrum (a) shows anodized PS while spectra (b), (c) and (d) show $5 \mathrm{~s}, 30 \mathrm{~s}$ and $60 \mathrm{~s}$ metallization times of PS surface in the solutions, respectively. The peaks shown in Fig. 3a are in good agreement with the data reported in the literature [2-8].
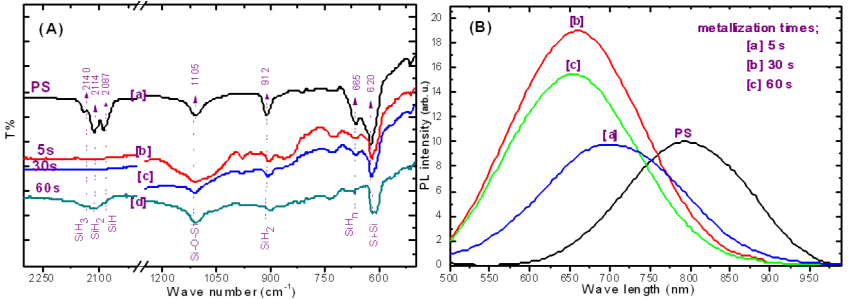

Fig. 3. (A) FTIR spectra of PS samples: (a) as-anodized, $(b),(c)$ and $(d)$ are metallized PS with $\mathrm{Na}$ in solutions containing $3 \mathrm{mM} \mathrm{NaNO}$ metal salt using immersing plating method for metallization time of 5 , 30 and $60 \mathrm{~s}$, respectively and (B) is their PL spectra compared with the as-anodized PS.

It can be seen from Fig. 3a that a significant change in the spectra was observed following the immersion of PS into the $3 \mathrm{mM} \mathrm{NaNO}_{3}$ metal salt containing an aqueous solution for various immersion times. The absorption peak centered around $1105 \mathrm{~cm}^{-1}$ is due to $\mathrm{Si}-\mathrm{O}-\mathrm{Si}$ stretching modes not significantly affected by immersion time of the PS in the solutions. The peaks of $\mathrm{Si}-\mathrm{H}_{n}$ at 2087, 2114 and $2140 \mathrm{~cm}^{-1}$ tend to disappear in the Na metallized samples. Furthermore, other $\mathrm{Si}-\mathrm{H}_{n}$ $(n=1$ and 2$)$ related peaks at 912 and $665 \mathrm{~cm}^{-1}$ have also decreased or disappeared for the metallized samples. The broadening and intensification in the $\mathrm{Si}-\mathrm{O}-\mathrm{Si}$ stretch band at $1105 \mathrm{~cm}^{-1}$ and the diminishing of $\mathrm{Si}-\mathrm{H}$ related peaks are due to the replacement of the hydrogen atoms by the oxygen and/or oxygen-Na metal atoms. Similar results were obtained by other metallizations ( $\mathrm{Li}$ and $\mathrm{K}$ ) [3].

The PL spectra shown in Fig. 3b were obtained from the as anodized samples and surfaces of the PS metallized with Na. After the metallization, a blue-shift of the in 
the PL spectra are observed. FWHM of the PL spectra of metallized samples increased when compared to the as anodized PS samples. Looking at the FTIR results, it can be observed that the metal deposition rate onto the PS surface is accompanied by the oxidation of Si surface. The deposition of the metal depends on the concentration of metal ions and immersion times [7]. It is possible that hydrogen atoms leaving the PS surface might have formed new oxide related interface states with surface modification. This oxygen or metal $(\mathrm{Na})$ oxide related electronic states should play an important role in the PL mechanism of the PS. The PL spectra support the results obtained with FTIR spectrum.

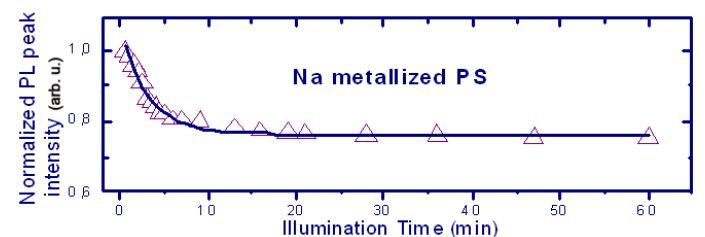

Fig. 4. Illumination time-dependence of normalized photoluminescence intensity of the metallized PS for $30 \mathrm{~s}$ (solid line is function fit).

The PS sample shown in Fig. 4 was metallized in $\mathrm{NaNO}_{3}$ solutions for $30 \mathrm{~s}$. There was a decrease in normalized luminescence intensity of $\mathrm{Na}$ metallized PS due to continuous exposure to the UV lamp for $60 \mathrm{~min}$ (Fig. 4). However, the decrease in the intensity of PL is only about $25 \%$ of the as anodized intensity. Therefore, it can be concluded that Na metallization of PS by immersion plating leads to a stable and efficient luminescence from the PS compared with as-anodized PS luminescence that was shown in Fig. 2.

\section{Conclusions}

Alkali metallization (only Na metallization results were shown for clarity) via immersion plating caused oxidation on the PS surface. The FTIR spectroscopy results made it clear that the PS surface oxygen and/or Na metal pairs are pacified after immersion plating. Therefore, newly formed oxygen or metal oxide related energy states give rise to a blue shift in the PL spectra. Similar results were obtained for other metallizations ( $\mathrm{Li}$ and $\mathrm{K}$ ).

The experimental results in this study suggest the possibility that surface modification with the alkali metallization by immersion plating is a relatively easy way for the oxidation of the PS surface, leading to a stable and efficient luminescence from the PS.

\section{References}

[1] A. Benilov, I. Gavrilchenko, I. Benilova, V. Skryshevsky, M. Cabrera, Sensors Actuators A 137, 245 (2007).

[2] E. Kayahan, Appl. Surf. Sci. 257, 4311 (2011).

[3] K. Esmer, E. Kayahan, Appl. Surf. Sci. 256, 1548 (2009).

[4] E. Kayahan, N. Ceylan, K. Esmer, Appl. Surf. Sci. 255, 2808 (2008).

[5] M.V. Wolkin, J. Jorne, P.M. Fauchet, G. Allan, C. Delerue, Phys. Rev. Lett. 82, 197 (1999).

[6] S.M. Hossain, C. Chakraborty, S.K. Dutta, J. Das, H. Saha, J. Lumin. 91, 195 (2000).

[7] F.A. Harraz, T. Sakka, Y.H. Ogata, Phys. Status Solidi A 197, 51 (2003).

[8] M. Fujiwara, T. Matsumoto, H. Kobayashi, K. Tanaka, N. Happo, K. Hori, J. Lumin. 113 , 243 (2005). 